\title{
Controlled Radical Polymerization of Vinyl Chloride Mediated by Xanthene-9-Thione
}

Peng Cui ${ }^{\text {a }}$, Changtong Song ${ }^{\mathrm{a}}$, Xianhong Zhang*a, Dong Chen ${ }^{\mathrm{a}}$, Yuhong Ma ${ }^{\mathrm{a}}$, Wantai

$$
\text { Yang*a,b }
$$

${ }^{a}$ Beijing Engineering Research Center of Syntheses and Applications of Waterborne Polymers and ${ }^{\mathrm{b}}$ State Key Laboratory of Chemical Resource Engineering, Beijing University of Chemical Technology, Beijing 100029, China.

* E-mail: xhzhang@mail.buct.edu.cn. Tel:18901078667 (X.Z.).

* E-mail: yangwt@mail.buct.edu.cn. Tel: +86 01064432262488 (W.Y.).

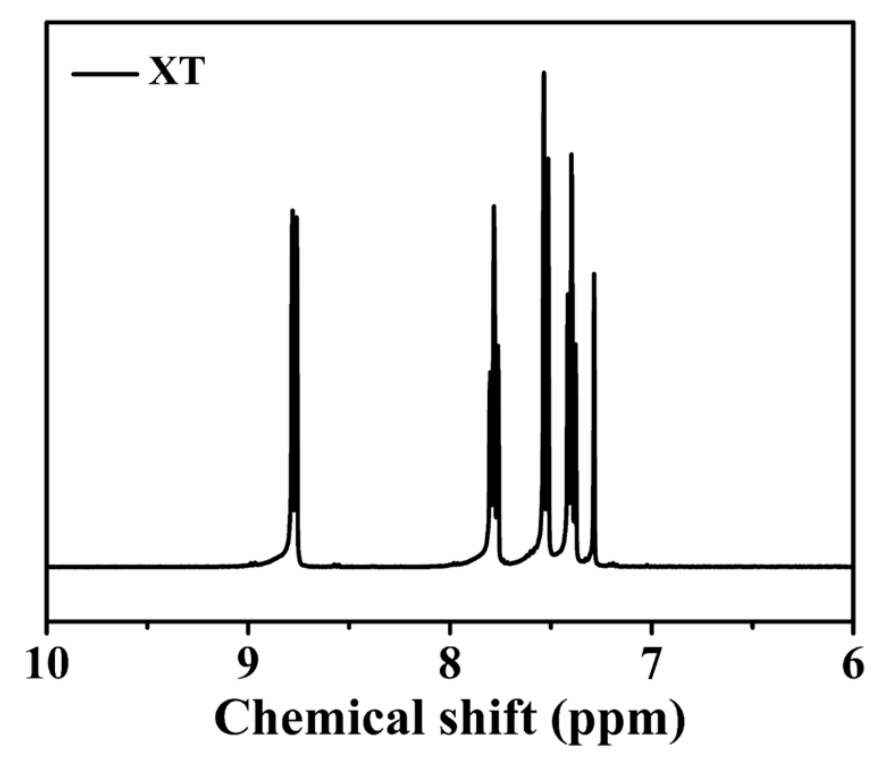

Figure S1. ${ }^{1} \mathrm{H}$ NMR spectroscopy of the XT 
Table S1. The effects of TBCP/XT molar ratios on monomer conversion and $M_{\mathrm{n}}$ at

\begin{tabular}{|c|c|c|c|c|c|}
\hline No. & $\begin{array}{c}\text { Molar ratio of } \\
\mathrm{TBCP} / \mathrm{XT}\end{array}$ & $\begin{array}{l}\text { Polymerization time } \\
\text { (h) }\end{array}$ & $\begin{array}{c}\text { Conversion } \\
(\%)\end{array}$ & $\begin{array}{c}M_{\mathrm{n}} \mathrm{GPC} \\
(\mathrm{Da})\end{array}$ & PDI \\
\hline 1 & $1.5: 1$ & 12 & 0 & - & - \\
\hline 2 & $1.5: 1$ & 24 & 0 & - & - \\
\hline 3 & $1.5: 1$ & 48 & 0 & - & - \\
\hline 4 & $1.5: 1$ & 72 & 0 & - & - \\
\hline 5 & $2: 1$ & 18 & 11 & 7100 & 1.96 \\
\hline 6 & $2: 1$ & 24 & 18.8 & 9000 & 2.09 \\
\hline 7 & $2: 1$ & 36 & 30.9 & 9700 & 2.05 \\
\hline 8 & $2: 1$ & 50 & 34.8 & 9900 & 2.4 \\
\hline 9 & $2: 1$ & 72 & 52.8 & 10300 & 2.04 \\
\hline 10 & $2.5: 1$ & 12 & 9.6 & 8200 & 1.81 \\
\hline 11 & $2.5: 1$ & 24 & 25.8 & 8900 & 2.00 \\
\hline 12 & $2.5: 1$ & 48 & 39.1 & 9200 & 1.98 \\
\hline 13 & $2.5: 1$ & 72 & 62 & 10000 & 2.03 \\
\hline 14 & $3: 1$ & 12 & 19.4 & 9200 & 1.92 \\
\hline 15 & $3: 1$ & 24 & 34.4 & 9400 & 1.91 \\
\hline 16 & $3: 1$ & 48 & 50.5 & 11800 & 2.15 \\
\hline 17 & $3: 1$ & 72 & 65.5 & 10600 & 2.14 \\
\hline 18 & $5: 1$ & 12 & 25 & 7300 & 2.00 \\
\hline 19 & $5: 1$ & 24 & 45.7 & 11300 & 2.88 \\
\hline 20 & $5: 1$ & 48 & 72.8 & 12200 & 3.39 \\
\hline 21 & $5: 1$ & 72 & 96.4 & 11000 & 3.56 \\
\hline
\end{tabular}



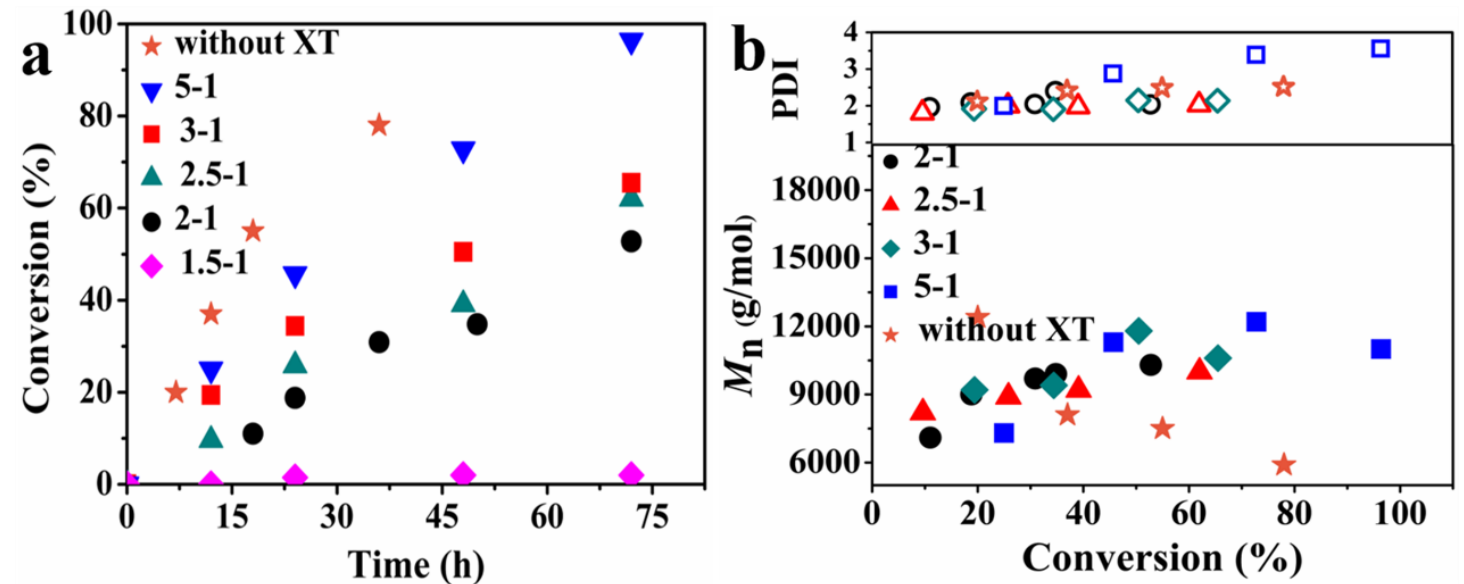

Figure S2. The effects of TBCP/XT molar ratios on the (a) monomer conversion and (b) $M_{\mathrm{n}}$ of PVC.

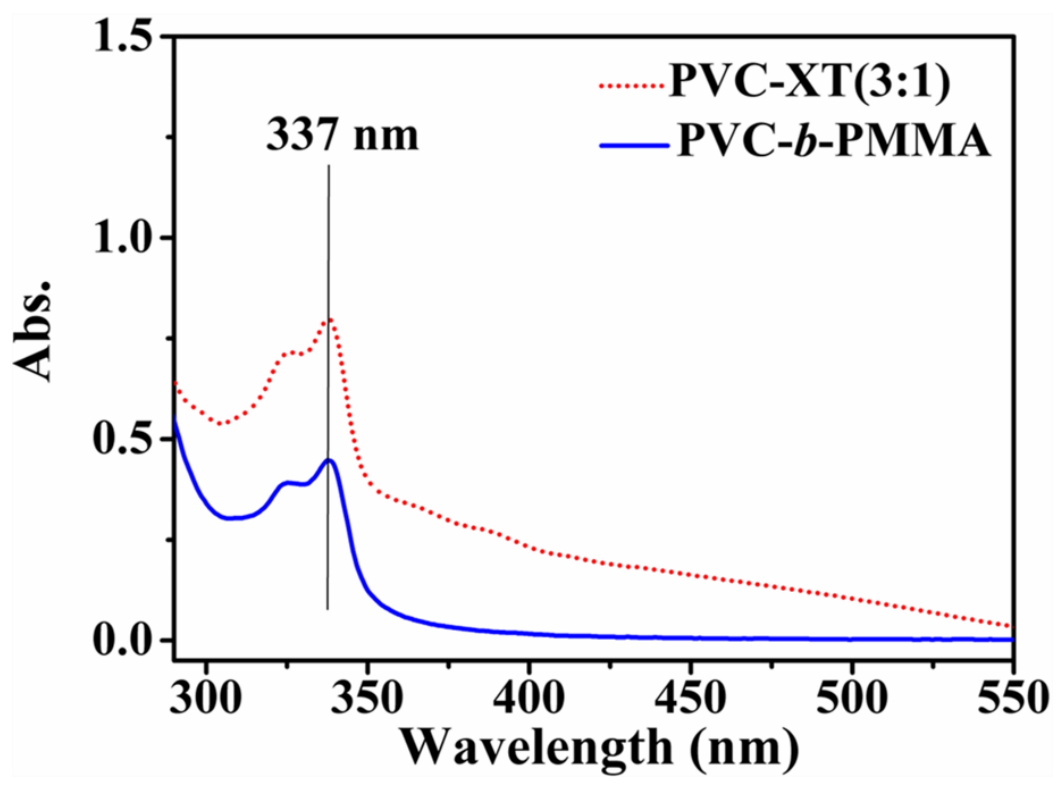

Figure S3. UV-vis spectra of PVC-XT and PVC- $b$-PMMA measured in THF 


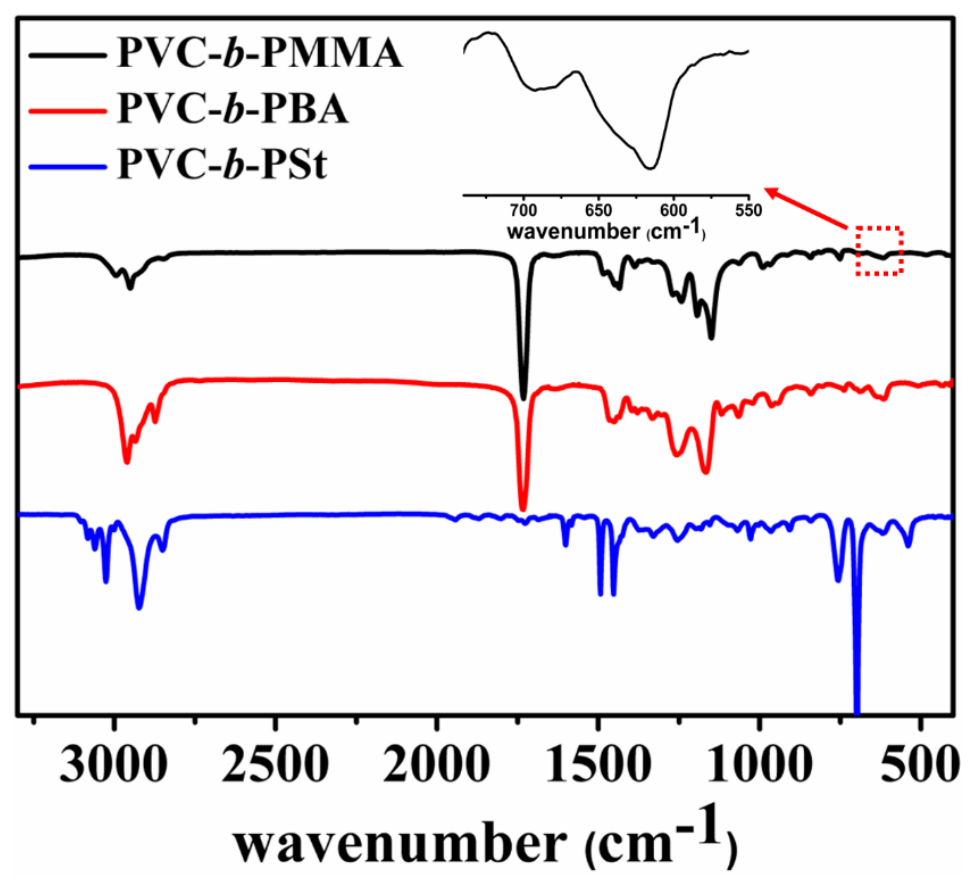

Figure S4. FT-IR spectra of the PVC based copolymers.

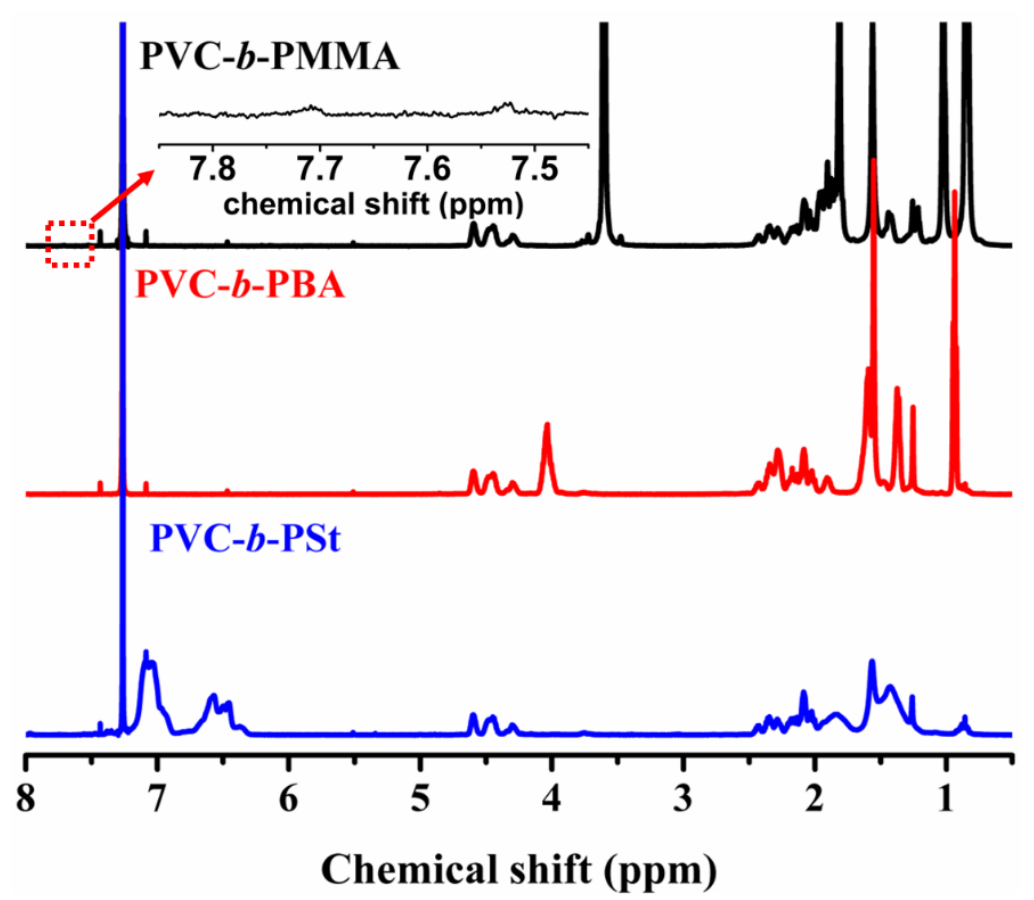

Figure S5. ${ }^{1} \mathrm{H}$ NMR spectra of the PVC based copolymers. 